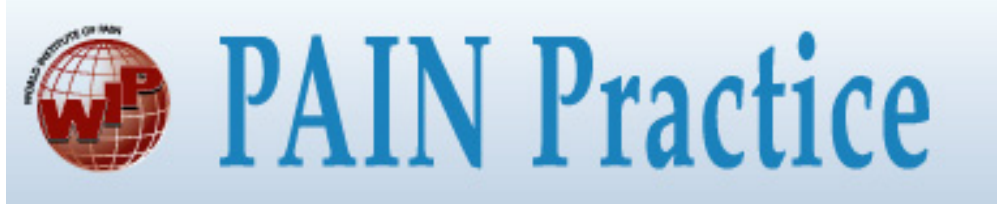

\title{
A Regions of Interest Voxel-based morphometry study of the human brain during high-frequency spinal cord stimulation in patients with Failed Back Surgery Syndrome
}

\begin{tabular}{|r|l|}
\hline Journal: & Pain Practice \\
\hline Manuscript ID & PPR-2020-0133.R1 \\
\hline Manuscript Type: & Original Manuscript \\
\hline Keywords: & $\begin{array}{l}\text { Spinal cord stimulation, neuroplasticity, structural brain alterations, } \\
\text { Regions of Interest Voxel-Based Morphometry }\end{array}$ \\
\hline \multicolumn{2}{|l}{} \\
\hline
\end{tabular}

\section{SCHOLARONE \\ Manuscripts}




\title{
A Regions of Interest Voxel-based morphometry study of the human brain during high- frequency spinal cord stimulation in patients with Failed Back Surgery Syndrome
}

\begin{abstract}
Introduction

The effectiveness of Spinal Cord Stimulation (SCS) as pain-relieving treatment for Failed Back Surgery Syndrome (FBSS) has already been demonstrated. However, potential structural and functional brain alterations resulting from subsensory SCS are less clear. The aim of this study is to test structural volumetric changes in a priori chosen regions of interest related to chronic pain after 1 month and 3 months of high-frequency SCS, in patients with FBSS.

Methods
\end{abstract}

Eleven patients with FBSS, scheduled for SCS implantation were included in this study. All patients underwent a magnetic resonance imaging protocol before SCS implantation, 1 month and 3 months after high frequency SCS. Pain intensity, pain catastrophizing and sleep quality were also measured. Regions of Interest Voxel-based morphometry was used to explore GM volumetric changes over time. Additionally, volumetric changes were correlated with changes in pain intensity, catastrophizing and sleep quality.

Results

Significant decreases were found in volume in the left and right hippocampus over time. More specifically, a significant difference was revealed between volumes before SCS implantation and after 3 months of SCS. Repeated measures correlations revealed a significant positive correlation between volumetric changes in the left hippocampus and changes in back pain score over time and between volumetric changes in the right hippocampus and changes in back pain score over time.

Conclusion 
In patients with FBSS, high-frequency SCS influences structural brain regions over time. The volume of the hippocampus was decreased bilaterally after 3 months of high-frequency SCS with a positive correlation with back pain intensity.

\section{Keywords}

Spinal Cord Stimulation; Neuroplasticity; Regions of Interest Voxel-Based Morphometry; Structural brain alterations. 


\section{Introduction}

Nowadays, Spinal Cord Stimulation (SCS) is a widely accepted treatment option for patients with Failed Back Surgery Syndrome (FBSS) ${ }^{1}$. SCS is considered to be cost-effective, more effective in obtaining pain relief and induces a higher quality of life compared to conventional treatment options 2. Initially, the success of SCS was allocated to paresthesia coverage to achieve a maximal overlap with the painful areas ${ }^{3}$. Several stimulation paradigms are now available with subthreshold stimulation at high frequencies and/or other stimulation paradigms which can induce equal or even greater pain relief than standard SCS 4,5 .

High-frequency SCS at $10 \mathrm{kHz}$ (HF-10 SCS) is such a new paradigm that does not elicit paresthesia, thereby proposed to eliminate the need to establish paresthesia mapping and coverage ${ }^{6}$. Despite the success, the exact working mechanisms of HF-10 SCS are still not completely unravelled. Basic research with computational models indicated that HF-10 SCS is primarily blocking large-diameter fibres, while recruiting medium- and smaller diameter fibres in combination with an inhibition of wide dynamic range neurons 7,8 . The hypotheses generated by the computer simulation studies 7 have not been supported by animal observations which instead indicate a very delayed, but direct inhibitory effect on dorsal horn neurons even with very low SCS amplitude ${ }^{9}$. Besides these segmental effects, supraspinal effects are suggested to play a role as well in the inhibitory effects of SCS ${ }^{10}$. Resting state fMRI previously demonstrated an increase in connectivity over time (until 3 months after HF-10 SCS) between the anterior insula and regions of the frontoparietal network and the central executive network ${ }^{11}$. Despite the changes in functional brain connectivity after HF-10 SCS, structural modifications due to HF-10 SCS are not yet explored. 
Neuroplasticity is the phenomenon by which the central nervous system can adapt to new experiences by changing its function and structure ${ }^{12}$. Kregel et al. (2015) revealed in their systematic review moderate but indistinct evidence for alterations in global and regional grey- and white matter in patients with chronic low back pain ${ }^{13}$, indicative for the occurrence of neuroplasticity in chronic pain patients.

Voxel-based morphometry is a technique that enables researchers to quantify grey matter and white matter on a voxel-by-voxel level. The goal of this study is to identify structural volumetric changes in specific regions known as to be involved in chronic pain between baseline and 3 months of HF-10 SCS. Additionally, we hypothesize that structural brain differences will occur simultaneously with changes in the clinical status of patients. Therefore, we will also evaluate whether there are correlations between structural brain differences and differences in clinical data namely pain intensity, pain catastrophizing and sleep quality.

\section{Methods \& materials}

\subsection{Participants}

Eleven consecutive FBSS patients (chronic, intractable pain of the trunk and/or limbs which has remained refractory to conservative therapy) who are eligible for HF-10 SCS, were recruited at XXX between January 2016 and July 2017. All patients were recruited before the SCS trial. Patients were not eligible if they had an existing extreme fear for entering MRI, general contraindications for MRI or a life expectancy less than 6 months. All participants provided written informed consent prior to participation. 
The study was approved by the ethics committee of the XXX and registered at ClinicalTrials.gov XXX. This study was conducted according to the revised declaration of Helsinki (1998).

\subsection{Study protocol}

In this prospective cohort study, patients were included before SCS implantation and followed up until 3 months of receiving HF-10 SCS. During this period, six additional individual appointments took place (Figure 1).

\subsubsection{Study visits}

The first two visits took place before SCS implantation. The remaining four visits were scheduled after permanent implantation. The first (V1), third (V3), and fifth (V5) visit were short appointments of about 20 minutes and were scheduled 2 weeks before the neuro-imaging visits (V2, V4, V6). During visits V1, V3, and V5, every subject received an Actiwatch spectrum plus (Phillips Respironics Inc., Murrysville, PA, USA) to collect objective sleep quality data and a Numeric Rating Scale (NRS) diary for pain intensity. During V2 (baseline), V4 (1 month after permanent HF-10 SCS) and V6 (3 months after permanent HF-10 SCS) patients were asked to complete two questionnaires (Pain Catastrophizing Scale [PCS] and Pittsburgh sleep quality index [PSQI]) and they underwent a neuroimaging MRI-protocol. Between the second and third visit, patients underwent a trial implantation of SCS, followed by a definitive implantation of the SCS system. According to the XXX reimbursement rules, a trial period of 4 weeks with an external neurostimulator should be provided with a pain reduction of at least $50 \%$ and a reduction in pain medication more than $50 \%$. In this study, all patients had a positive trial, where after they all received a definitive implantation.

\subsubsection{Implantation/programming procedures}

Two percutaneous leads were placed in the posterior spinal epidural space under radiographic imaging and attached to either an external stimulator (during the trial phase) or a subcutaneously implanted pulse generator (IPG). The distal tip of one lead was placed at T8, whereas a second lead 
tip was placed at T9, both near anatomical midline. All patients received two percutaneous leads. Stimulation was configured bipolarly in all patients. All patients included in this study were implanted with a Senza rechargeable system (Nevro Corp., Redwood City, CA, USA). All patients received paresthesia-free SCS, which was delivered with a pulse width of $30 \mu \mathrm{sec}$ in combination with a frequency of $10,000 \mathrm{~Hz}$ and amplitudes between 1.5 and $2.5 \mathrm{~mA}$. The variation of amplitudes was defined based on an algorithm. This algorithm entails a rigid protocol varying from 0 to $3.5 \mathrm{~mA}$ that is starting with $2.5 \mathrm{~mA}$. Patients had the liberty to change the amplitude with steps of $0.5 \mathrm{~mA}$ in function of individual pain relief during the first two weeks. Afterwards, during the entire follow-up period, the stimulation parameters of the permanent IPG remained unchanged.

\subsection{Outcome measurements}

\subsubsection{Questionnaires:}

Patients were asked to fill in a NRS diary three times a day namely in the morning, noon and evening, for a period of two weeks to evaluate pain intensity. All participants rated their leg and back pain intensity separately. A score of zero represents no pain, whereas a score of ten represents the worst imaginable pain. Afterwards, all scores were summed and averaged (separately for back and leg pain).

Pain catastrophizing was evaluated with the PCS. This questionnaire, consisting of 13 questions, measures three different aspects namely pain magnification, rumination and helplessness. All questions are evaluating past painful experiences and are scored on a five point scale, ranging from zero (not at all) to four (all the time). The resulting total score ranges from 0-52 with a cut-off value of 30 for a clinical relevant degree of catastrophizing. The validity of the questionnaire is considered good, with an internal consistency ranging between 0.6 and $0.87^{14-16}$.

For collecting objective sleep quality parameters, the Actiwatch Spectrum Plus (Philips Respironics, 
Inc., Murrysville, PA, USA) was used for a period of two weeks. This device contains an accelerometer and color sensitive photodiodes to analyze circadian rhythms and sleep-wake patterns. Five sleep quality parameters were evaluated: wake after sleep onset percentage, sleep onset latency, mean night-time activity, sleep efficiency and number of wake bouts. Actigraphy is considered to be an objective tool for estimating sleep parameters in low back pain (LBP) patients and has proven to be a valid tool to analyze sleep quality after only one week of measurements ${ }^{17,18}$.

Subjective sleep quality was measured with the Pittsburgh Sleep Quality Index (PSQI). This questionnaire collects information about the usual sleep habits of the past month. The PSQI consists of 19 questions and 5 questions related to the partner, each with a scoring range of 0 (no difficulties during the last month) to 3 (difficulties more than three times a week) points. Only the 19 questions filled in by the patient, were used to calculate the total score. Scores are categorized to form seven component scores (sleep latency, sleep quality, sleep duration, use of sleep medication, daytime dysfunctioning, sleep disturbances and habitual sleep efficiency) with a total output score varying from 0 to 21. A recent meta-analysis concluded that the PSQI has a strong reliability and validity ${ }^{19}$.

\subsubsection{Structural brain alterations:}

Structural brain changes between baseline, 1 month of HF-10 SCS and 3 months of HF-10 SCS were evaluated by means of the Voxel-Based Morphometry Diffeomorphic Anatomical Registration Through Exponentiated Lie (VBM-DARTEL) toolbox in Statistical Parametric Mapping (SPM12), implemented in Matlab 2018b (Mathworks ${ }^{\mathrm{TM}}$ Boston) ${ }^{20-22}$. VBM-DARTEL is an MRI-based neuroimaging technique which involves a voxel-wise comparison of the volume of GM regions of interest (Rol's) between study visits ${ }^{23}$. This method enables the identification of volumetric changes in local brain regions. More specifically, the following regions were investigated: bilateral hippocampus, thalamus, dorsolateral prefrontal cortex, ventromedial prefrontal cortex, anterior cingulate, posterior cingulate, insular regions and parietal lobe ${ }^{24}$. 


\subsubsection{MRI data acquisition}

MRI scans were performed on a GE MR 750w Discovery 3T using a 24-channel head coil. A high resolution anatomical image was acquired using an axial fast spoiled gradient echo (FSPGR) bravo scan, consisting of 124 axial slices with slice thickness $1 \mathrm{~mm}$; no inter-slice gap; $\mathrm{TR}=7.74 \mathrm{~ms} ; \mathrm{TE}=$ $3.75 \mathrm{~ms} ;$ flip angle $=12^{\circ} ;$ scan matrix $=256 \times 256 ;$ FOV $=240 \times 240 \mathrm{~mm}^{2}$. Patients were instructed to stay awake and to immediately inform the investigators in case any unusual sensation was felt at the implantation site, because SCS was left on during imaging. The introduction of MRI-conditioned SCS devices enables exploration of MOA of SCS, not only during trial period but also on long-term implanted devices.

\subsubsection{Image processing}

Regional Rol volumes were assessed with the VBM-DARTEL procedure of Ashburner and Friston ${ }^{20,21 .}$ The DARTEL algorithm was selected to evaluate the Rol volumes more accurately. DARTEL-based VBM provides more precise inter-subject alignment in association with image registration compared to standard VBM and optimized VBM techniques ${ }^{25}$. After screening for gross anatomical abnormalities, reorientation of the images was manually set to the anterior commissure.

In first instance raw data (DICOM format) was converted into a NIFTI format. Thereupon, image preprocessing started with segmentation of the individual brains into GM, WM and cerebrospinal fluid (CSF) maps. Only GM maps were used for registration in the analysis ${ }^{26}$. These maps contain the proportion of GM volume in a voxel. To improve the accuracy of the normalization step, a DARTEL non-linear image registration procedure was performed as a second step ${ }^{21,27,28}$. A template was generated based on the individual tissue maps from all patients. All individual tissue maps were matched to determine the nonlinear deformations for warping all the GM images so that they matched each other to a group template from their own mean ${ }^{21,29}$. Next, the GM images were nonlinearly normalized onto the DARTEL template and transformed to the Montreal Neurological 
Institute (MNI) coordinate space ${ }^{29,} 30$. The normalized tissue maps were modulated by multiplying the voxel values with the Jacobian determinants of the deformation field to represent GM volume proportion maps and to correct for local expansions ${ }^{31}$. Finally, images were smoothed with an isotropic Gaussian kernel $8 \mathrm{~mm}$ full width at half maximum (FWHM) for having more accurately aligned images ${ }^{32}$. Statistical analysis was done using the smoothed, modulated, DARTEL- warped and normalized GM modalities. Before performing further analyses, a screening for segmentation or normalization errors was executed by visual inspection of the smoothed GM volume maps.

To identify volume alterations over time, a general linear model was created in SPM 12. Smoothed GM pre-processed segments were entered into a one-way ANOVA to investigate volume variances in the Rol's on a voxel-by-voxel based method. The following Rol's were identified: bilateral hippocampus, thalamus, dorsolateral prefrontal cortex, ventromedial prefrontal cortex, anterior cingulate, posterior cingulate, insular regions and parietal lobe ${ }^{24}$ using the Neuromorphometrics atlas in SPM (http://Neuromorphometrics.com).

For avoiding edge effects, an absolute mask threshold of 0.2 was selected, meaning that all brain volumes should have at least a GM proportion of $0.2^{32}$. Age was selected as covariate to correct for unwanted morphological variations. A voxel significance threshold of $p<0.001$ and a cluster threshold of 25 voxels was selected. After defining each SPM cluster as a Rol mask with the MarsBar Toolbox version 0.44 (http://marsbar.sourceforge.net) ${ }^{33}$, volumes were extracted. This was performed for all significant voxels within each SPM cluster.

The anatomical location of GM volumetric changes was identified with the Automated Anatomical Labelling Atlas (http://www.gin.cnrs.fr/en/tools/aal/) by means of MRIcron (https://www.nitrc.org/projects/mricron). To precisely define the anatomical structure(s) out of which our ROI's were composed from, a clustered labelling procedure was executed in the AAL3 toolbox, implemented in SPM ${ }^{34}$.

\section{$\underline{\text { 2.3.5 Statistical analysis }}$}


Statistical analyses were performed with the Statistical Package for the Social Sciences Software (IBM SPSS for Windows, version 25, SPSS Inc., Chicago, Illinois, USA). Friedman tests with post-hoc Wilcoxon tests were calculated to analyze differences in volumetric changes and to analyze differences in total scores on the questionnaires, between the three visits. Repeated measures correlations between Rol volumes and total score on questionnaires were computed in R Studio (version 1.2 .5001 ) to evaluate correlation at the longitudinal level ${ }^{35}$. Only questionnaires with a significant difference between the visits were used in the correlation analysis. The significance level was set at 0.05. A Bonferroni correction was applied to correct for multiple comparisons.

\section{Results}

\subsection{Demographics}

Eleven patients with FBSS were included in this study ( 2 males, 9 females) with a median age of 56[Q1:50-Q3:57]. All patients previously underwent at least 1 spinal surgery and 6 patients already underwent 2 or more spinal surgeries. Most of the patients ( 8 out of 11 patients) experienced pain for at least 3 years. Nine patients experienced a neuropathic pain component, measured as a score of at least 4/10 on the "Douleur Neuropathique" questionnaire (DN4). Patient characteristics are presented in Table 1.

\subsection{Clinical Results}

A significant decrease in NRS score for back pain $\left(\chi^{2}=9.53, p=0.009\right)$ and leg pain $\left(\chi^{2}=6.2, p=0.045\right)$ 
was observed over time. At baseline, the median NRS score for back pain was 5.89 (Q1-Q3: 4.47 7.6), at 1 month 4.19 (Q1-Q3: $2.94-4.42$ ) and at 3 months of HF-10 SCS 3.41 (Q1-Q3: $2.51-4.46$ ). Wilcoxon tests revealed a significant difference between baseline and 1 month $(Z=-2.6, p=0.009)$ and between baseline and 3 months $(Z=-2.6, p=0.009)$. For leg pain, the median NRS score at baseline was 5.85 (Q1-Q3: $3.59-7.07$ ), at 1 month 3.5 (Q1-Q3: $2.43-5.38$ ) and at 3 months of HF-10 SCS 4.21 (Q1-Q3: $3.43-5$ ). Wilcoxon tests revealed a significant difference between baseline and 3 months of SCS $(Z=-2.09, p=0.037)$. No significant differences were revealed for PCS scores $\left(\chi^{2}=6\right.$, $p=0.05)$, PSQI scores $\left(\chi^{2}=2.89, p=0.24\right)$ nor for parameters of the actiwatch.

\subsection{Brain volume measurements}

All patients were compliant with the neuroimaging protocols.

Significantly lower GM volumes were observed over time in the left (MNI coordinates $-12,-38,-2$; voxel size 118) and right (MNI coordinates 20, -36, 6; voxel size 46) hippocampus (Figure 2). More specifically, a significant difference was revealed between volumes before SCS implantation and after 3 months of SCS for the left hippocampus $(t=4.67, p<0.001)$ and right hippocampus $(t=3.98$, $\mathrm{p}<0.001)$. No differences were detected between the volumes before implantation and after 1 month of SCS, nor between the volumes at 1 month and 3 months of SCS. The volume of the left hippocampus decreased from $0.398 \mathrm{~mL}$ (Q1-Q3: $0.393-0.436 \mathrm{~mL}$ ) at baseline, to $0.381 \mathrm{~mL}$ (Q1-Q3: $0.346-0.401 \mathrm{~mL}$ ) after 1 month and $0.372 \mathrm{~mL}(\mathrm{Q} 1-\mathrm{Q} 3: 0.363-0.399 \mathrm{~mL}$ ) at 3 months of HF-10 SCS. In the right hippocampus, the volume decreased from $0.405 \mathrm{~mL}$ (Q1-Q3: $0.382-0.440 \mathrm{~mL}$ ) at baseline, to $0.389 \mathrm{~mL}$ (Q1-Q3: $0.364-0.418 \mathrm{~mL}$ ) after 1 month and $0.374 \mathrm{~mL}$ (Q1-Q3: $0.354-0.395$ $\mathrm{mL})$ at 3 months of HF-10 SCS. 


\subsection{Correlation between clinical outcomes and brain volumetric measurements}

Only NRS back pain scores and leg pain scores were statistically significant over time and were considered for the correlation analysis with WM volume and GM volume alterations. At the intraindividual (i.e. longitudinal) level, a statistically significant positive correlation was observed between the GM volumes in the left hippocampus and pain intensity scores for back pain $(r=0.747, p<0.001)$. This means that at the intra-individual level a decrease in NRS pain intensity score is correlated with a decrease in volume in the hippocampus. Additionally, a significant positive correlation was observed between the GM volumes in the right hippocampus and pain intensity scores for back pain at the longitudinal level $(r=0.669, p<0.001)$ (Figure 3).

\section{Discussion}

Paresthesia-free spinal cord stimulation at $10 \mathrm{kHz}$ or High Frequency SCS distinguishes itself from Low Frequency or standard SCS (LF-SCS) by several specific characteristics and variables. By definition and in strong contrast, HF-10 SCS generates pain relief using $10 \mathrm{kHz}$ without paresthesia, while LF-SCS induces paresthesia at a frequency $<200 \mathrm{~Hz}^{36}$. Besides the electrical characteristics, the mechanism of action seems to differ as well. While LF-SCS activates at the segmental level largediameter fibers, producing paresthesia, HF-10 SCS might enhance an inversion of large and smaller diameter fiber thresholds. This produces a therapeutic window in which smaller fibers are recruited while larger ones are inactivated ${ }^{36}$. Several other hypotheses have been posted in order to explain the mechanism of action of HF-10 SCS, such as a reversible depolarization blockade, desynchronization of neural signals and membrane integration ${ }^{9}$. Summarizing, converging lines of evidence support the direct effect of HF-10 SCS on local dorsal structures in the spinal cord. 
Although, there is a relative absence of literature evaluating the supraspinal mechanisms of HF-10 SCS ${ }^{37}$.

Evaluation of the supraspinal effects of SCS by neuroimaging might include measuring functional interferences, biochemical interactions and structural adaptations ${ }^{38}$. Voxel-based morphometry, an automated technique, uses statistical tests across all voxels in T1-weighted volumetric MRI images in order to identify volumetric differences between groups ${ }^{25}$. In the past, grey and white matter analyses have been used to identify structural changes in several chronic pain conditions, among which low back pain ${ }^{13,39-42}$. Nevertheless, there is a lack of evidence towards investigating therapeutic effects on the supraspinal structures in low back pain. In this study, based on an a priori hypothesis, we investigated for the first time the supraspinal structures during HF-10 SCS over time in patients with FBSS, in specific Rol's involved in chronic pain conditions ${ }^{24}$. This so called Rol VBM analysis is more sensitive than a whole brain VBM and specifically useful for the evaluation of an a priori hypothesis ${ }^{43,44}$.

This study revealed a bilateral decrease of the GM volume in the hippocampus after 3 months of HF10 SCS. Reductions in hippocampal GM have previously been demonstrated in different chronic pain populations ${ }^{45-47}$. Moreover, these results are in line with the study of Luchtmann et al. (2015) which demonstrated a decreased hippocampal volume in patients suffering from chronic low back pain after microsurgical lumbar discectomy ${ }^{48}$. It might be suggested that treatment could reverse the previously occurred neuroplastic changes due to the presence of chronic pain. This idea was supported by several studies in which important similarities between pain chronification and the learning mechanism, coordinated by the hippocampus, were mentioned ${ }^{49-59}$. The hippocampus is involved in learning processes whereby it is considered the primary brain structure for storage and retrieval of long-term explicit memories ${ }^{60}$. In 2012, Mutso et al. redefined chronic pain in terms of context conditioning and extinction. They considered chronic pain as a state of continuous learning whereby aversive emotional associations are constantly made with otherwise incidental events because of the persistent chronic pain state ${ }^{53}$. This leads to the persistence of pain-related 
memories and/or the inability of the extinction of pain memories $39,45,48,52-56,61-63$. Ultimately, structural and functional brain alterations, among which several hippocampal abnormalities, result in the common comorbidities as observed in chronic pain disorders such as cognitive impairment, deterioration of long-and short term-memory and the inability to extinct pain memories $49,52,55,57,58$.

Other than its function in learning mechanisms and the memory, recent studies also suggested that the hippocampal formation plays a key role in the modulation of pain $49,55,56$. Additionally, the hippocampus is also involved in the contextual and emotional processing of pain $53,55,56,58,64,65$. Based on these findings, it is possible that the observed decrease in grey matter volume in the hippocampus may in fact imply a normalisation of the hippocampal function. As such, the reduced volume in the hippocampus in our study might be a surrogate of perceived recovery after treating FBSS patients with HF-10 SCS. This hypothesis could be further substantiated by the positive correlation between pain intensity and brain volume. More specifically, a decrease in NRS back pain intensity score was correlated with a decrease in volume in the hippocampus. No correlation was found between leg pain intensity and volume in the hippocampus. A possible explanation could be found in the underlying distribution of leg pain intensity which is not fully suggestive for a linear time trend. Future studies are needed to further elucidate the correlation at the intra-individual level.

One of the strengths of this study is the implementation of the DARTEL protocol for the normalisation of the anatomical images, insuring more precise results for subsequent volumetric analyses. Another strength of this study is the use of an a priori ROI VBM analysis, which reduces the risk of type I errors, in contrast to a whole-brain VBM analysis ${ }^{66}$. Besides the hypothesis driven design, this study also correlated clinical outcome parameters with supra-spinal effects, giving an important additional insight in the temporal aspect of neuroplasticity during HF-10 SCS. Besides the transparent analysis scheme and the consistency in statistical analyses, this study also has some limitations. By definition, an important limitation of this pilot study is the limited sample size. Furthermore, patients were not asked to refrain from their daily medication due to ethical reasons. 
Specifically on the hippocampus, chronic administration of gabapentin and carbamazepine can increase neurodegenerative changes in animal models ${ }^{67}$. Additionally, opioids are suggested to be associated with hippocampus-dependent learning and memory ${ }^{68}$. It might be possible that medication use has influenced our results. However, direct effects on the hippocampus have not been previously explored wherefore future studies are necessary to evaluate the exact role of medication on this type of studies ${ }^{47}$. Concerning pain intensity scores, a pain diary was used in this study to obtain more accurate pain intensity scores and limit recall bias. However, this choice makes it rather difficult to compare the obtained pain relief with other studies ${ }^{5}$. Finally, the clusters undergoing a volumetric decrease were not corrected for family wise errors. However, strict $\mathrm{p}$ values were applied in all analyses.

\section{Conclusion}

Summing up, this Rol VBM study revealed for the first time that HF-10 SCS in patients with FBSS influences structural brain architecture over time. Bilaterally, the hippocampal volume appears to be decreased after 3 months of HF-10 SCS and is positively correlated with back pain intensity. 


\section{References}

1. Frey ME, Manchikanti L, Benyamin RM, Schultz DM, Smith HS, Cohen SP. Spinal cord stimulation for patients with failed back surgery syndrome: a systematic review. Pain physician. 2009;12:379-397.

2. Grider JS, Manchikanti L, Carayannopoulos A, Sharma ML, Balog CC, Harned ME, et al. Effectiveness of Spinal Cord Stimulation in Chronic Spinal Pain: A Systematic Review. Pain physician. 2016;19:E33-54.

3. Alo KM, Holsheimer J. New trends in neuromodulation for the management of neuropathic pain. Neurosurgery. 2002;50:690-703; discussion 703-694.

4. Morales A, Yong RJ, Kaye AD, Urman RD. Spinal Cord Stimulation: Comparing Traditional Low-frequency Tonic Waveforms to Novel High Frequency and Burst Stimulation for the Treatment of Chronic Low Back Pain. Curr Pain Headache Rep. 2019;23:25.

5. Kapural L, Yu C, Doust MW, Gliner BE, Vallejo R, Sitzman BT, et al. Novel 10-kHz Highfrequency Therapy (HF10 Therapy) Is Superior to Traditional Low-frequency Spinal Cord Stimulation for the Treatment of Chronic Back and Leg Pain: The SENZA-RCT Randomized Controlled Trial. Anesthesiology. 2015;123:851-860.

6. Amirdelfan K, Vallejo R, Benyamin R, Yu C, Yang T, Bundschu R, et al. High-Frequency Spinal Cord Stimulation at $10 \mathrm{kHz}$ for the Treatment of Combined Neck and Arm Pain: Results From a Prospective Multicenter Study. Neurosurgery. 2019.

7. Arle JE, Mei L, Carlson KW, Shils JL. High-Frequency Stimulation of Dorsal Column Axons: Potential Underlying Mechanism of Paresthesia-Free Neuropathic Pain Relief. Neuromodulation. 2016;19:385-397.

8. Lempka SF, McIntyre CC, Kilgore KL, Machado AG. Computational analysis of kilohertz frequency spinal cord stimulation for chronic pain management. Anesthesiology. 2015;122:13621376.

9. Linderoth B, Foreman RD. Conventional and Novel Spinal Stimulation Algorithms: Hypothetical Mechanisms of Action and Comments on Outcomes. Neuromodulation. 2017;20:525533.

10. Sivanesan E, Maher DP, Raja SN, Linderoth B, Guan Y. Supraspinal Mechanisms of Spinal Cord Stimulation for Modulation of Pain: Five Decades of Research and Prospects for the Future. Anesthesiology. 2019;130:651-665.

11. De Groote S, Goudman L, Peeters R, Linderoth B, Vanschuerbeek P, Sunaert S, et al. Magnetic Resonance Imaging Exploration of the Human Brain During $10 \mathrm{kHz}$ Spinal Cord Stimulation for Failed Back Surgery Syndrome: A Resting State Functional Magnetic Resonance Imaging Study. Neuromodulation. 2019.

12. Westlake KP, Byl NN. Neural plasticity and implications for hand rehabilitation after neurological insult. J Hand Ther. 2013;26:87-92; quiz 93.

13. Kregel J, Meeus M, Malfliet A, Dolphens M, Danneels L, Nijs J, et al. Structural and functional brain abnormalities in chronic low back pain: A systematic review. Seminars in arthritis and rheumatism. 2015;45:229-237.

14. Micheal S. The Pain Catastrophizing Scale. 1995. 
15. Osman A, Barrios FX, Kopper BA, Hauptmann W, Jones J, O'Neill E. Factor structure, reliability, and validity of the Pain Catastrophizing Scale. Journal of behavioral medicine. 1997;20:589-605.

16. Cheng ST, Chen PP, Chow YF, Chung JWY, Law ACB, Lee JSW, et al. The Pain Catastrophizing Scale-short form: psychometric properties and threshold for identifying high-risk individuals. International psychogeriatrics. 2019:1-10.

17. Alsaadi SM, McAuley JH, Hush JM, Bartlett DJ, Henschke N, Grunstein RR, et al. Detecting insomnia in patients with low back pain: accuracy of four self-report sleep measures. BMC musculoskeletal disorders. 2013;14:196.

18. Van de Water AT, Holmes A, Hurley DA. Objective measurements of sleep for non-laboratory settings as alternatives to polysomnography--a systematic review. Journal of sleep research. 2011;20:183-200.

19. Mollayeva T, Thurairajah P, Burton K, Mollayeva S, Shapiro CM, Colantonio A. The Pittsburgh sleep quality index as a screening tool for sleep dysfunction in clinical and non-clinical samples: A systematic review and meta-analysis. Sleep medicine reviews. 2016;25:52-73.

20. Ashburner J, Friston KJ. Voxel-based morphometry--the methods. Neuroimage. 2000;11:805821.

21. Ashburner J. A fast diffeomorphic image registration algorithm. Neuroimage. 2007;38:95-

113.

22. Mechelli A, Price C, Friston K, Ashburner J. Voxel-Based Morphometry of the Human Brain: Methods and Applications. Vol. 1: Current Medical Imaging Reviews; 2005.

23. Michael AM, Evans E, Moore GJ. Influence of Group on Individual Subject Maps in SPM Voxel Based Morphometry. Front Neurosci. 2016;10:522.

24. Levins KJ, Drago T, Roman E, Martin A, King R, Murphy P, et al. Magnetic resonance spectroscopy across chronic pain disorders: a systematic review protocol synthesising anatomical and metabolite findings in chronic pain patients. Syst Rev. 2019;8:338.

25. Whitwell JL. Voxel-based morphometry: an automated technique for assessing structural changes in the brain. The Journal of neuroscience : the official journal of the Society for Neuroscience. 2009;29:9661-9664.

26. Ashburner J, Friston KJ. Unified segmentation. Neuroimage. 2005;26:839-851.

27. Matsuda H, Mizumura S, Nemoto K, Yamashita F, Imabayashi E, Sato N, et al. Automatic voxel-based morphometry of structural MRI by SPM8 plus diffeomorphic anatomic registration through exponentiated lie algebra improves the diagnosis of probable Alzheimer Disease. AJNR Am J Neuroradiol. 2012;33:1109-1114.

28. Pereira JM, Xiong L, Acosta-Cabronero J, Pengas G, Williams GB, Nestor PJ. Registration accuracy for $\mathrm{VBM}$ studies varies according to region and degenerative disease grouping. Neuroimage. 2010;49:2205-2215.

29. Mechelli A, Price CJ, Friston KJ, Ashburner J. Voxel-Based Morphometry of the Human Brain: Methods and Applications. Curr. Med. Imaging Rev. 2005;1.

30. Ashburner J. Computational anatomy with the SPM software. Magnetic resonance imaging. 2009;27:1163-1174.

31. Van Schuerbeek P, Baeken C, De Raedt R, De Mey J, Luypaert R. Individual differences in local gray and white matter volumes reflect differences in temperament and character: a voxel-based morphometry study in healthy young females. Brain Res. 2011;1371:32-42.

32. Van Schuerbeek P, Baeken C, De Mey J. The Heterogeneity in Retrieved Relations between the Personality Trait 'Harm Avoidance' and Gray Matter Volumes Due to Variations in the VBM and ROI Labeling Processing Settings. PloS one. 2016;11:e0153865.

33. Brett M, Anton J-L, Valabregue R, Poline J-B. Region of interest analysis using an SPM toolbox. 8th International Conference on Functional Mapping of the Human Brain. Sendia, Japan2002.

34. Rolls ET, Huang CC, Lin CP, Feng J, Joliot M. Automated anatomical labelling atlas 3. Neuroimage. 2019:116189.

35. Bakdash JZ, Marusich LR. Repeated Measures Correlation. Front Psychol. 2017;8:456. 
36. Arle JE, Mei L, Carlson KW. Fiber Threshold Accommodation as a Mechanism of Burst and High-Frequency Spinal Cord Stimulation. Neuromodulation. 2019.

37. Caylor J, Reddy R, Yin S, Cui C, Huang M, Huang C, et al. Spinal cord stimulation in chronic pain: evidence and theory for mechanisms of action. Bioelectron Med. 2019;5.

38. Lee M, Tracey I. Neuro-genetics of persistent pain. Curr Opin Neurobiol. 2013;23:127-132.

39. Mao C, Wei L, Zhang Q, Liao X, Yang X, Zhang M. Differences in brain structure in patients with distinct sites of chronic pain: A voxel-based morphometric analysis. Neural regeneration research. 2013;8:2981-2990.

40. Wood PB. Variations in brain gray matter associated with chronic pain. Current rheumatology reports. 2010;12:462-469.

41. Schmidt-Wilcke T, Leinisch E, Ganssbauer S, Draganski B, Bogdahn U, Altmeppen J, et al. Affective components and intensity of pain correlate with structural differences in gray matter in chronic back pain patients. Pain. 2006;125:89-97.

42. Buckalew N, Haut MW, Morrow L, Weiner D. Chronic pain is associated with brain volume loss in older adults: preliminary evidence. Pain medicine (Malden, Mass.). 2008;9:240-248.

43. Seidman L, Biederman J, Liang L, Valera EM, Monuteaux MC, Brown A, et al. Gray matter alterations in adults with attention-deficit/hyperactivity disorder identified by voxel based morphometry. Biol Psychiatry. 2011;69:857-866.

44. Cao H, Wang R, Luo X, Li X, Hallett M, Thompson-Westra J, et al. A Voxel-Based Magnetic Resonance Imaging Morphometric Study of Cerebral and Cerebellar Gray Matter in Patients Under 65 Years with Essential Tremor. Med Sci Monit. 2018;24:3127-3135.

45. Baliki MN, Schnitzer TJ, Bauer WR, Apkarian AV. Brain morphological signatures for chronic pain. PloS one. 2011;6:e26010.

46. Hayes DJ, Chen DQ, Zhong J, Lin A, Behan B, Walker M, et al. Affective Circuitry Alterations in Patients with Trigeminal Neuralgia. Front Neuroanat. 2017;11:73.

47. Vaculik MF, Noorani A, Hung PS, Hodaie M. Selective hippocampal subfield volume reductions in classic trigeminal neuralgia. Neuroimage Clin. 2019;23:101911.

48. Luchtmann M, Baecke S, Steinecke $Y$, Bernarding J, Tempelmann C, Ragert $P$, et al. Changes in gray matter volume after microsurgical lumbar discectomy: a longitudinal analysis. Frontiers in human neuroscience. 2015;9:12.

49. McCarberg B, Peppin J. Pain Pathways and Nervous System Plasticity: Learning and Memory in Pain. Pain Medicine. 2019.

50. Price TJ, Inyang KE. Commonalities between pain and memory mechanisms and their meaning for understanding chronic pain. Prog Mol Biol Trans/ Sci. 2015;131:409-434.

51. Apkarian AV, Mutso AA, Centeno MV, Kan L, Wu M, Levinstein M, et al. Role of adult hippocampal neurogenesis in persistent pain. Pain. 2016;157:418-428.

52. Mutso AA, Petre B, Huang L, Baliki MN, Torbey S, Herrmann KM, et al. Reorganization of hippocampal functional connectivity with transition to chronic back pain. J Neurophysiol.

2014;111:1065-1076.

53. Mutso AA, Radzicki D, Baliki MN, Huang L, Banisadr G, Centeno MV, et al. Abnormalities in hippocampal functioning with persistent pain. The Journal of neuroscience : the official journal of the Society for Neuroscience. 2012;32:5747-5756.

54. Khan SA, Keaser ML, Meiller TF, Seminowicz DA. Altered structure and function in the hippocampus and medial prefrontal cortex in patients with burning mouth syndrome. Pain. 2014;155:1472-1480.

55. Thompson JM, Neugebauer V. Cortico-limbic pain mechanisms. Neurosci Lett. 2019;702:15-

23.

56. Chong CD, Dumkrieger GM, Schwedt TJ. Structural Co-Variance Patterns in Migraine: A Cross-Sectional Study Exploring the Role of the Hippocampus. Headache. 2017;57:1522-1531.

57. Berger SE, Vachon-Presseau É, Abdullah TB, Baria AT, Schnitzer TJ, Apkarian AV. Hippocampal morphology mediates biased memories of chronic pain. Neuroimage. 2018;166:86-98. 58. McEwen BS. Plasticity of the hippocampus: adaptation to chronic stress and allostatic load. Ann N Y Acad Sci. 2001;933:265-277. 
59. Liu MG, Chen J. Roles of the hippocampal formation in pain information processing. Neurosci Bull. 2009;25:237-266.

60. Phillips RG, LeDoux JE. Differential contribution of amygdala and hippocampus to cued and contextual fear conditioning. Behav Neurosci. 1992;106:274-285.

61. Yuan C, Shi H, Pan P, Dai Z, Zhong J, Ma H, et al. Gray Matter Abnormalities Associated With Chronic Back Pain: A Meta-Analysis of Voxel-based Morphometric Studies. Clin J Pain. 2017;33:983990.

62. Smallwood RF, Laird AR, Ramage AE, Parkinson AL, Lewis J, Clauw DJ, et al. Structural brain anomalies and chronic pain: a quantitative meta-analysis of gray matter volume. The journal of pain : official journal of the American Pain Society. 2013;14:663-675.

63. Maleki N, Becerra L, Brawn J, McEwen B, Burstein R, Borsook D. Common hippocampal structural and functional changes in migraine. Brain Struct Funct. 2013;218:903-912.

64. Ziv $\mathrm{M}$, Tomer R, Defrin $\mathrm{R}$, Hendler $\mathrm{T}$. Individual sensitivity to pain expectancy is related to differential activation of the hippocampus and amygdala. Hum Brain Mapp. 2010;31:326-338.

65. Borsook D, Becerra L, Hargreaves R. Biomarkers for chronic pain and analgesia. Part 2: how, where, and what to look for using functional imaging. Discov Med. 2011;11:209-219.

66. Vanneste S, Van De Heyning P, De Ridder D. Tinnitus: a large VBM-EEG correlational study. PloS one. 2015;10:e0115122.

67. Olaibi OK, Osuntokun OS, ljomone OM. Effects of chronic administration of gabapentin and carbamazepine on the histomorphology of the hippocampus and striatum. Ann Neurosci.

2014;21:57-61.

68. Kutlu MG, Gould TJ. Effects of drugs of abuse on hippocampal plasticity and hippocampusdependent learning and memory: contributions to development and maintenance of addiction.

Learn Mem. 2016;23:515-533. 


\section{Tables}

\begin{tabular}{|c|c|c|c|c|c|c|c|}
\hline Pt & Sex & $\begin{array}{l}\text { Age at } \\
\text { implantation } \\
\text { (years) }\end{array}$ & $\begin{array}{l}\text { Pain } \\
\text { duration } \\
\text { (years) }\end{array}$ & $\begin{array}{l}\text { Number of } \\
\text { previous } \\
\text { surgeries }\end{array}$ & $\begin{array}{l}\text { DN4 } \\
\text { score }\end{array}$ & $\begin{array}{l}\text { Pain medication } \\
\text { before } \\
\text { implantation }\end{array}$ & $\begin{array}{l}\text { Pain } \\
\text { medication } \\
\text { after } \\
\text { implantation }\end{array}$ \\
\hline 1 & $M$ & 57 & 14 & 3 & 6 & $\begin{array}{l}\text { Opioids + } \\
\text { pregabaline }\end{array}$ & Opioids \\
\hline 2 & $\mathrm{~F}$ & 56 & 5 & 2 & 4 & $\begin{array}{l}\text { Opioids + } \\
\text { pregabaline }\end{array}$ & Opioids \\
\hline 3 & $\mathrm{~F}$ & 67 & 1 & 1 & 5 & $\begin{array}{l}\text { Opioids + } \\
\text { pregabaline }\end{array}$ & Opioids \\
\hline 4 & $M$ & 57 & 1 & 1 & 6 & $\begin{array}{l}\text { Opioids + } \\
\text { pregabaline }\end{array}$ & $\begin{array}{l}\text { Opioids }+ \\
\text { paracetamol }\end{array}$ \\
\hline 5 & $\mathrm{~F}$ & 46 & 5 & 3 & 4 & $\begin{array}{l}\text { Opioids + } \\
\text { pregabaline }\end{array}$ & $\begin{array}{l}\text { Paracetamol + } \\
\text { opioids if } \\
\text { necessary }\end{array}$ \\
\hline 6 & $\mathrm{~F}$ & 53 & 3 & 1 & 5 & $\begin{array}{l}\text { Opioids + } \\
\text { pregabaline }\end{array}$ & Opioids \\
\hline 7 & $\mathrm{~F}$ & 46 & 3 & 1 & 8 & $\begin{array}{l}\text { Opioids + } \\
\text { pregabaline }\end{array}$ & Opioids \\
\hline 8 & $\mathrm{~F}$ & 56 & 3 & 2 & 5 & None & Paracetamol \\
\hline 9 & $\mathrm{~F}$ & 59 & 6 & 5 & 2 & $\begin{array}{l}\text { Weak opioids + } \\
\text { pregabaline }\end{array}$ & 1 \\
\hline 10 & $\mathrm{~F}$ & 50 & 1 & 1 & 8 & $\begin{array}{l}\text { Weak opioids + } \\
\text { pregabaline }\end{array}$ & NSAID \\
\hline 11 & $\mathrm{~F}$ & 55 & 8 & 2 & 2 & $\begin{array}{l}\text { Weak opioids + } \\
\text { pregabaline }\end{array}$ & $\begin{array}{l}\text { NSAID if } \\
\text { necessary }\end{array}$ \\
\hline $\begin{array}{l}\text { Summary } \\
\text { statistics }\end{array}$ & $\begin{array}{l}\text { Males: } 2 \\
(18.2 \%) \\
\text { Females: } 9 \\
(81.8 \%)\end{array}$ & $56(50-57)$ & $3(2-5.5)$ & $2(1-2.5)$ & $5(4-6)$ & & \\
\hline
\end{tabular}

Table 1. Individual patient characteristics of the $\mathbf{1 1}$ patients that are included in this study. The last

row is presenting the summary statistics with the median value and corresponding quartiles for all

patients or the absolute number and percentages in case of categorical data. Abbreviations. DN4:

Douleur Neuropathique 4 questionnaire, F:female, M:male, pt:patient. 


\section{Figures}

Figure 1. Flow chart of the study protocol. In total, the study consisted of six study visits whereby each neuroimaging study was preceded by a shorter visit two weeks before the neuroimaging visit, to complete questionnaires and provide the Actiwatch to patients. Abbreviations. AW: Actiwatch, HF-10 SCS: high frequency spinal cord stimulation, Q: questionnaires, V: visit.

Figure 2. Grey matter volume alterations between baseline and HF-10 SCS after 3 months in patients with FBSS. Significant grey matter alterations were found in the left and right hippocampus ( $p<0.001$, uncorrected). The colour bar represents the t-value of the GM volume analysis whereby all blue colours refer to a decrease in volume. Abbreviations. A: , L:left, R:right.

Figure 3. Repeated measures correlation between volume of left hippocampus (left) and right (right) hippocampus and average back pain intensity for all patients. At the intra-individual (i.e. longitudinal) level, a statistically significant positive correlation was observed between pain intensity scores for back pain and the GM volumes in the left hippocampus $(r=0.747, p<0.001)$ (left) and right hippocampus ( $r=0.669, p<0.001)$ (right). The blue line is presenting the datapoints obtained on baseline, the green line the datapoints collected after 1 months HF-10 SCS and the red line the datapoints after 3 months of HF-10 SCS. The green lines between the time points are presenting the direction of the observed correlation. Starting from the baseline observations, the correlation 
demonstrates that at the intra-individual level, a decrease in NRS pain intensity score is correlated with a decrease in volume in the hippocampus. 


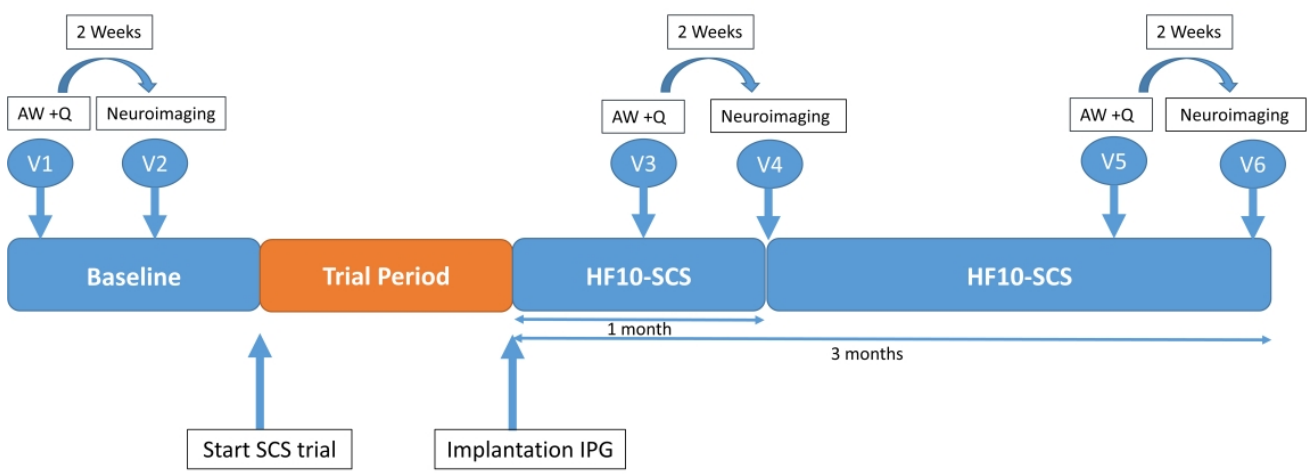

Figure 1

$338 \times 190 \mathrm{~mm}(600 \times 600$ DPI $)$

\section{Pain Practice}




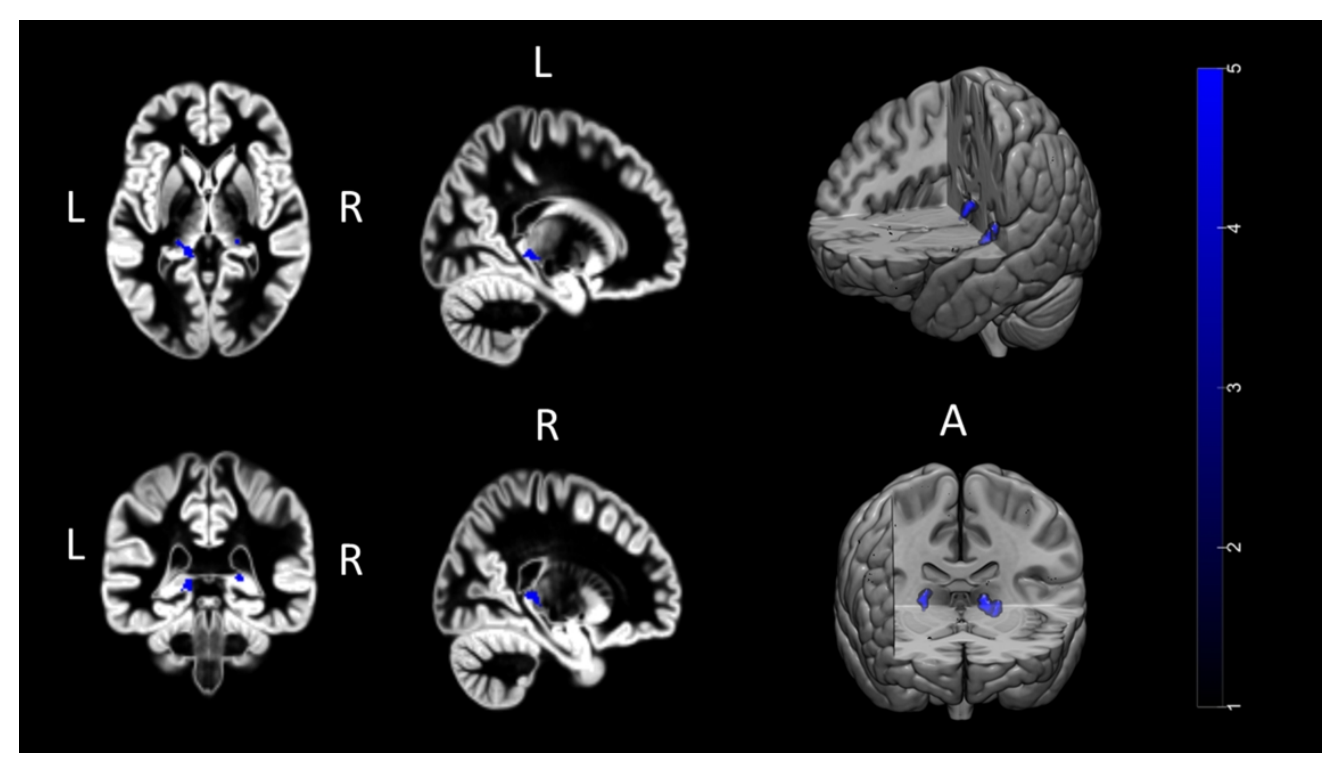

Figure 2

$338 \times 190 \mathrm{~mm}(300 \times 300$ DPI $)$ 

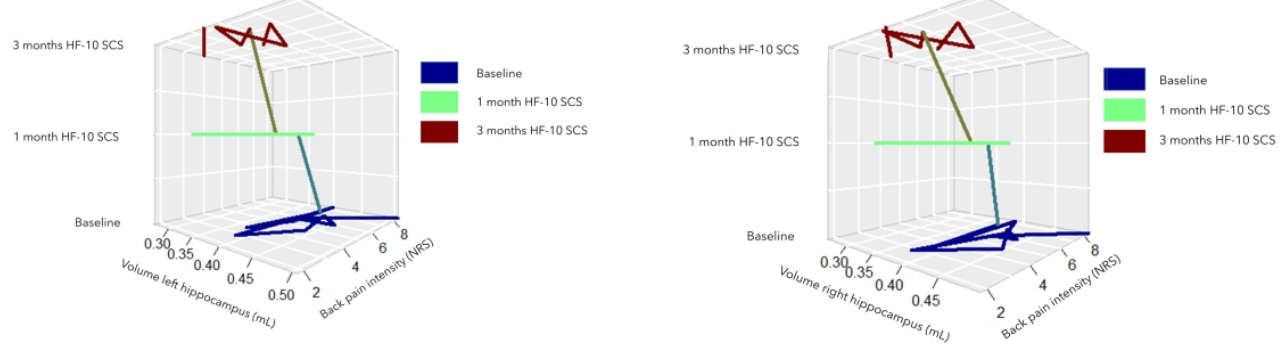

Figure 3

$338 \times 190 \mathrm{~mm}(300 \times 300$ DPI $)$ 